\title{
New approach to catheterization of the heart in infants and children
}

\author{
G. Azzolina, S. A. Eufrate, and A. Allella \\ From the Department of Paediatric Cardiothoracic Surgery, Ospedale Generale Provinciale, Massa, Italy
}

The peripheral blood vessels of infants and children are difficult to cannulate and to repair. The lumen will accommodate only small bore catheters and these are harder to manoeuvre, and impair the quality of angiograms. Lengthening of the procedure and an increase in the amount of contrast medium which is needed both contribute to the morbidity. To our knowledge, cannulation of the internal jugular vein and/or carotid artery for purpose of heart catheterization in humans has never been reported. The technique of this approach is described in detail and illustrated. A brief outline of the advantages and disadvantages of the procedure is given. The fact that the technique requires an operator experienced in handling major vessels is stressed. In all, 705 approaches to the great vessels of the neck have now been performed, and both the potential complications and those encountered are listed. With strict adherence to and familiarity with the technique the procedure is safe and offers, we believe, decided advantages.

Cannulation of arteries and veins for cardiac catheterization in the adult is usually easy. In the newborn and infants, however, it may be very difficult and time consuming. In the first two months of life mortality rates are very high, of the order of 6 per cent (Burchell and Ongley, 1968). The duration of the procedure is directly related to the mortality and morbidity in this category of patient (Banyai and Gordon, 1966), and the investigation should, in general, seek only those data essential to an accurate diagnosis. Because of the patient's size, difficulties are posed by the length-gauge ratio of the catheter and the amount of dye in relation to body weight to be delivered in unit of time (Banyai and Gordon, I966).

The technique we describe was developed between 1965 and 1966 by one of us (G.A.) and it has proved to be useful in the newborn, in infants, and in children up to the age of 3 to 4 years. We have often used it in children between 4 and 7 years of age, and occasionally in young adults. The approach is strongly indicated when previous catheterizations or other procedures have been carried out at or in the vicinity of the usual areas of venous or arterial cannulation. This is often the case in our patient population.

To our knowledge open cannulation of the internal jugular vein and/or the common carotid artery

Received 20 November 1972. for purpose of cardiac catheterization in humans has never been reported. From 1967 to 1971 inclusive we used the cervical approach in about 700 cases.

The object of this report is to illustrate in detail the new approach and to present its advantages in both cardiac catheterization and selective angiocardiography.

\section{Technique}

Venous cannulation (internal jugular vein)

After suitable preanaesthetic medication, the fasting patient is placed on the $x$-ray table in the supine position, with a small roll of cotton transversely under the shoulder blades and given an intravenous injection of sodium hydroxybutyrate, $60 \mathrm{mg} / \mathrm{kg}$ body weight.

Both arms are abducted 90 to 120 degrees, the forearms flexed about 45 degrees on the arms at the elbow, and the upper extremities fixed to the table with adhesive tape. The head is turned to the right. The patient is suitably painted and draped. Xylocaine ( $1 \%$ ) local anaesthesia is carried out at the site of planned incision. This is made $2.5 \mathrm{~cm}$ long, transversely placed on the left side of the neck 1.5 to $2 \mathrm{~cm}$ above the sternoclavicular joint and overlying the two heads of the sternocleidomastoid muscle (Fig. I). Exposure of progressively deeper layers is obtained using two small vein retractors. Blunt separation of the muscle fibres (two heads) of the sternomastoid is done with small scissors, going downwards and cranially at an angle of 45 degrees from the horizontal. The homohyoid muscle, when encountered, 


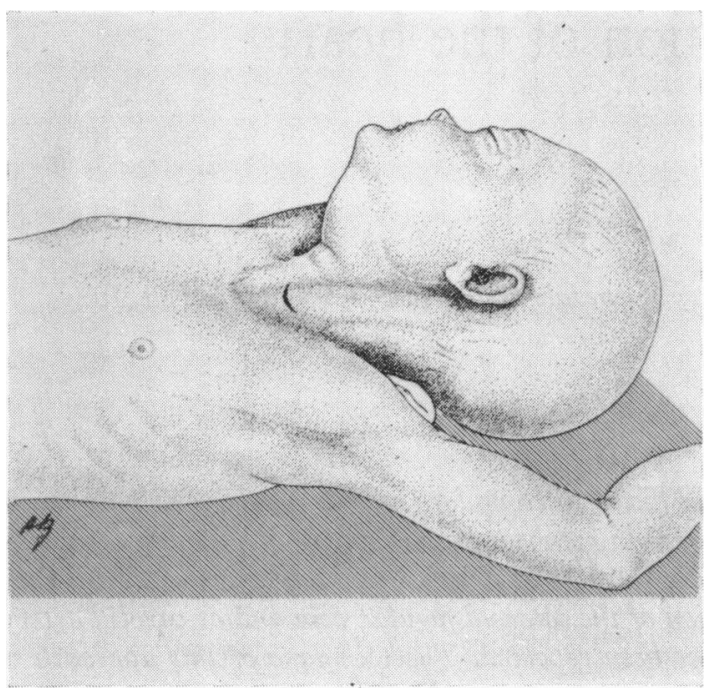

FIG. I The patient positioned for a left cervical approach. The location of the incision is shown.

is retracted. The internal jugular vein is found just below this level, picked up with vascular forceps, and cleared of adventitial tissue by blunt dissection. A small right angle clamp is used to isolate the vein in its medial and posterior aspects. Using this instrument, a suitable length of vein is freed. Two lengths of rubber band are passed under the internal jugular vein, one cephalad and one caudad, and secured at their ends with a straight mosquito clamp. Traction of them brings a length of vein out of the wound and controls it at the same time. An elliptical, longitudinal purse string suture of five zero vascular silk is placed in the adventitial-muscular layer of the internal jugular vein (Fig. 2). A longitudinal incision large enough to allow introduction of the catheter is made with a No. II knife blade within the area encompassed by the purse-string suture (Fig. 3). The vein should be kept filled with blood throughout. With the help of fine vascular thumb forceps, the tip of the catheter, suitably filled with normal saline and connected to a Luer-lock syringe similarly filled, is introduced in the lumen of the vein. The proximal rubber band control is now released enough to allow passage of the catheter and retightened to avoid spillage of blood but not enough to hinder manipulation of the catheter. The two rubber bands are now closed down on the vein by clamping them with the mosquito clamps near the vein (Fig. 4). The proximity of the common carotid artery, which lies medial and posterior to the internal jugular vein, allows for easy sampling of arterial blood.

Once the procedure is completed the catheter is withdrawn, the purse-string suture tied, and the rubber band controls removed. If the purse-string suture is properly applied and the adventitia is properly stripped from this area only minor narrowing of the vessels results. The skin is closed with two or three interrupted four zero vertical mattress sutures, and dressing is applied.

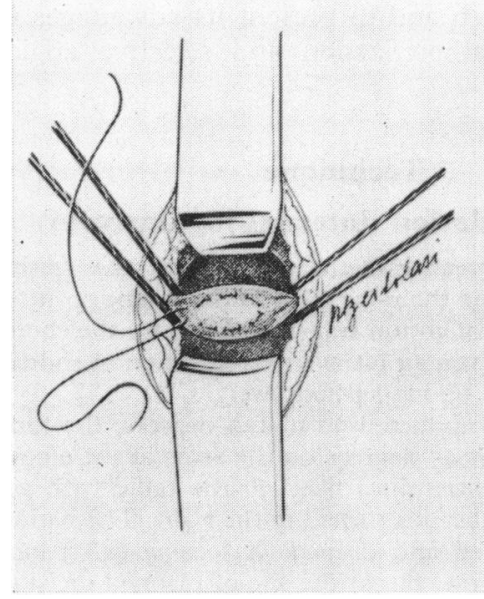

Fig. 2

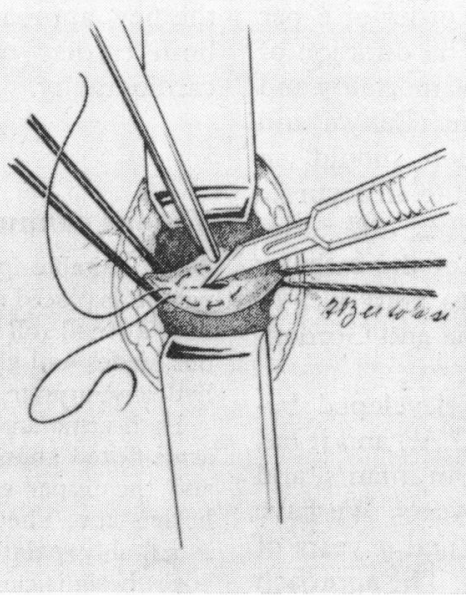

Fig. 3

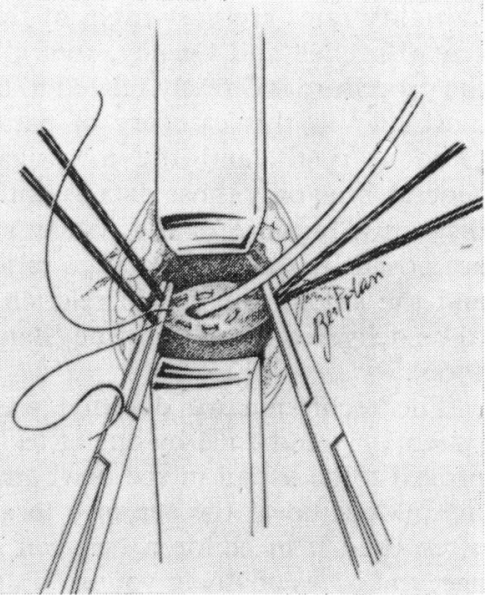

Fig. 4

FIG. 2 The sternocleidomastoid muscle has been retracted and held outside the field by two retractors. The internal jugular vein has been brought out of the wound and controlled by traction on the two controls (rubber band). The purse-string suture has been completed.

FIG. 3 The incision in the vein wall.

FIG. 4 The catheter has been introduced. The two rubber bands have been clamped with two straight mosquito clamps close to the vein to avoid bleeding during catheterization. 


\section{Arterial cannulation (common carotid artery)}

The technique for left heart catheterization using the common carotid artery is basically the same. The internal jugular vein is retracted laterally, and the artery is controlled with two loops of No. 2 black silk instead of rubber bands. The purse-string suture introduced in the adventitial-muscular layer is smaller than its venous counterpart. From time to time the pull on the two controls is released, allowing the artery to return to its normal position. The two heavy controls are not tightened down over the artery but used only to pull the artery out of the incision when necessary for manipulation and insertion of the catheter. In this way blood flow through the common carotid artery during catheterization is not seriously reduced. Blood loss is prevented by making an arteriotomy which is small enough to be completely filled by the catheter.

\section{Comments}

Technical points to be borne in mind for the successful accomplishment of the procedure are as follows. The skin incision must be accurately placed; the muscle heads must be separated going directly down toward the carotid sheath. If the separation is made in the wrong place the dissection will lead either too medially and anteriorly or too posteriorly; this may result in bleeding or other complications. Ligature of the internal jugular vein can be carried out with impunity should it become necessary. Blunt dissection of the vein (or artery) must be carried out below the adventitial layer which is stripped from the vein. This ensures that the dissection is carried out in the proper plane and facilitates the siting of the purse-string suture. The left internal jugular vein is usually cannulated and catheterization initiated within five minutes from the time of the skin incision.

The main advantages of this technique are: (I) there is considerable saving in time which is essential in the severely ill infant and newborn (Banyai and Gordon, 1966) and is preferable in children; (2) there is no restriction in availability of a suitable vein and artery; (3) there is constancy of presence and position of structures that are large and therefore easily identified; (4) the vessel does not have to be tied at the end of the procedure, so that it can be reused in the future; (5) because of the proximity to the major vessels and heart there is no need for long catheters; (6) the vessels are large and do not restrict the choice of catheter size; (7) absence of arterial spasm; (8) the proximity of the incisions to the heart makes it easier to place the catheter into and within the various chambers. Exploration of the pulmonary artery, in particular, is facilitated; (9) there is ease of, and speedy entrance into the left ventricle in any circumstances and the proximal aorta in coarctation; (I0) there is no arterial insufficiency after catheterization (no loss of radial pulse); (II) there is certain identification of persistent left superior vena cava when present; (I2) the larger size and short length of the catheter allow for injection of a smaller amount of contrast material in a shorter time (compact bolus), thus better pictures are obtained with less risk of dye toxicity or reactions. Excellent pictures may be obtained in babies around $3 \mathrm{~kg}$ body weight by injecting $1.5 \mathrm{ml}$ dye/kg body weight $(4.5-5 \mathrm{ml})$ at $30 \mathrm{ml} / \mathrm{sec}$ through a $50 \mathrm{~cm}$ long No. 7 N.I.H. catheter.

Potential hazards with this procedure are: laceration of the vein during dissection; injury to the vagus nerve, to the stellate ganglion, and to the thoracic duct; air embolism, pneumomediastinum, and pneumothorax. However, in our hands complications have been extremely rare, minor, and usually occurred while members of our group were becoming familiar with the procedure. Between 1967 and the end of 1971 615 open cannulations of the internal jugular vein (606 on the left and 9 on the right) and 90 cannulations of the common carotid artery ( 89 on the left and $I$ on the right) have been carried out, for a total of 715 approaches to the great vessels of the neck. We have had no cases of air embolism, or injury to the vagus nerve or the thoracic duct. We have seen one case of temporary Horner's syndrome (due to dissection carried too posteriorly) and four cases of pneumothorax, one of which was bilateral, after a left cervical approach. Pneumomediastinum occurs occasionally, but it is usually of no consequence; in general, it results from an incision too near the clavicle and a dissection too near the thoracic inlet. Vein lacerations may be due either to manipulative enlargement of the original incision, or incision of the posterior wall of the vein by the tip of the No. I I knife blade. They are extremely rare if precise technique is adhered to. We feel, therefore, that most of the possible complications are potential and avoidable rather than real, provided the described technique is strictly adhered to. Similarly we have never observed even temporary neurological or arterial complications from cannulation of the common carotid artery.

One objection to this approach is that balloon septostomy, as described by Rashkind, cannot be performed. In these cases we use the saphenofemoral route.

We fully recognize that the approach to the great vessels of the neck requires the presence of a qualified operator such as a surgeon or a cardiologist with considerable experience in handling vessels. These requirements may not be met in some cardiac laboratories where the assistance of, or training with, a surgical team is not readily available. Our cardiolo- 
gists, with whom we work in the strictest co-operation, now perform the cervical approach as we do. The technique requires an almost surgical level of skill if the really dangerous possible complications are to be avoided. The operator inexperienced in handling major vessels should not try this approach unless properly assisted.

We have not found that a larger catheter (No. 7 N.I.H.) is a source of greater myocardial irritability (Zimmerman, 1966) or is unduly rigid and dangerous (Banyai and Gordon, I966). In infants, however, we usually employ a No. 6 N.I.H.

We have often performed a second approach to the vessel of the neck from a few days to several months after the first procedure, and found normal appearing tissues and vessels, the only evidence of a previous cannulation being the silk purse-string knot. Up to one week soft oedema makes a second dissection fairly easy; from ro days to 3 weeks indurated oedema, which later disappears, makes dissection difficult, and during this period a second approach in the same area is not advisable.
The same approach may be used on the right side of the neck, as in cases of mirror-image dextrocardia. In cases of persistent left superior vena cava, which are always discovered with this technique, catheterization has usually been performed with more patience and caution and with some limitation, but in most cases completed in spite of some difficulty. If the procedure cannot be completed because of this situation, the catheter is withdrawn and the approach repeated on the right side.

\section{References}

Banyai, A. L., and Gordon, B. L. (1966). Advances in Cardiopulmonary Disease, vol. 3. Year Book Medical Publishers, Chicago.

Burchell, H. B., and Ongley, P. A. (1968). Editorial. The supplement on complications of cardiac catheterization. Circulation, 37, 675.

Zimmerman, H. A. (1966). Intravascular Catheterization, 2nd ed. Charles C. Thomas, Springfield, Illinois.

Requests for reprints to Dr. S. A. Eufrate, Department of Pediatric Cardiothoracic Surgery, Ospedale Generale Provinciale, 54100 Massa, Italy.

\section{Erratum}

The Editorial 'Understanding the Atrial Sound', by Bethell and Nixon, 1973, volume 35 , page 230 , contained a misprint. The third line of the first column should read 'is an inaudible vibration'. 